\title{
Overwhelming post-splenectomy infection with group B Streptococcus
}

\author{
O. C. Finnegan \\ M.B., M.R.C.P.
}

\author{
P. M. HAWKeY \\ B.Sc., M.B.
}

Bristol Royal Infirmary, Bristol BS2 $8 H W$

\section{Summary}

A case of overwhelming post-splenectomy infection is described. This occurred 10 years after splenectomy in an otherwise normal male adult. The infecting organism, $\beta$-haemolytic Streptococcus Group B, is usually of low pathogenicity, but in this case caused profound hypotension, jaundice and the shock lung syndrome. Overwhelming post-splenectomy infection with this organism has not previously been reported.

\section{Introduction}

It is acknowledged that there is a definite risk of overwhelming post-splenectomy infection (OPSI) in children, particularly when the spleen has been removed in the first 3 years of life. There has, however, been some disagreement on the hazards of splenectomy in normal adults. A case is reported of OPSI caused by an unusual micro-organism: a $\beta$ haemolytic Streptococcus of Lancefield's Group B ( $S$. agalactiae).

\section{Case report}

A 29-year-old labourer was admitted with a short history of headache, abdominal pain and vomiting. He had previously been well, and had been at work on the morning of admission. A splenectomy had been performed 10 years previously for traumatic splenic rupture. On admission he was flushed, pyrexial $\left(38.5^{\circ} \mathrm{C}\right)$ and slightly dehydrated, with a pulse of $105 / \mathrm{min}$ and BP of $140 / 90 \mathrm{mmHg}$. Examination of his chest was normal, but there was mild generalized abdominal tenderness. There was an episode of profuse watery diarrhoea during the examination. Investigations included $\mathrm{Hb} 14.0 \mathrm{~g} / \mathrm{dl}$, WCC $5.4 \times 10^{9} / 1$, plasma urea $6.4 \mathrm{mmol} / \mathrm{l}$ and normal plasma electrolytes.

He was rehydrated intravenously and treated symptomatically for his diarrhoea, but the following morning he experienced a rigor and was found to be dyspnoeic, cyanosed and confused. His pulse and $\mathrm{BP}$ were $140 / \mathrm{min}$ and $100 / 60 \mathrm{mmHg}$ respectively and there were scattered coarse crackles throughout of both lungs. The WCC had risen to $30 \times 10^{\%} / 1$ and the plasma urea to $13.8 \mathrm{mmol} / \mathrm{l}$. Serum bilirubin was of $34 \mu \mathrm{mol} / \mathrm{l}$, alkaline phosphatase $21 \cdot 3$ i.u./l, and $\dot{N}$ aspartate amino transferase 50 i.u./l. He was hyper- $\stackrel{\mathrm{N}}{\circ}$ ventilating but hypoxic with an arterial $\mathrm{PO}_{2}$ of $7.2 \mathrm{kPa}(54.0 \mathrm{mmHg})$ and $P \mathrm{CO}_{2}$ of $3.6 \mathrm{kPa}(27.0$ $\mathrm{mmHg}$ ). His BP fell to $70 / 40 \mathrm{mmHg}$, but responded $\$ to dopamine and steroids. Large volumes of i.v. crystalloids were required to restore his central $\stackrel{\rho}{\supset}$ venous pressure to normal. The chest X-ray showe $\overrightarrow{0}$ features consistent with the shock lung syndrome

Blood cultures yielded a heavy growth of a haemolytic Streptococcus of Lancefield's Group sensitive to benzyl penicillin, erythromycin, chloramphenicol and cefuroxime, and resistant to tetracycline. Lumbar puncture produced clear $\stackrel{\circ}{\varnothing}$ cerebrospinal fluid with no cells, and normal glucose and protein concentrations. It was sterile on culture and the organism was not isolated from any other site. As there was an unsubstantiated history of penicillin allergy, he was given i.v. chloramphenicol one $g$ thrice daily changed after 2 days to cefuroxime $1.5 \mathrm{~g}$ thrice daily.

Over the next 3 days his BP rose spontaneously and his urinary output was satisfactory, although he became jaundiced and remained hypoxic despite high concentrations of inspired oxygen. Thereafter he made a gradual but complete recovery, his radiology, biochemistry, and pulmonary function returning to normal within 3 weeks.

\section{Discussion}

The spleen has an important role in the body's $N$ defence against infection. It is involved in the $\omega$ elimination of particulate matter such as bacteria from the blood stream, and in the early production $\stackrel{O}{=}$ of antibody to circulating antigen. Despite the $\stackrel{\mathbb{D}}{\mathscr{N}}$ definite risks of OPSI in children, there are conflicting views on the hazards of infection in normal adults who require splenectomy after trauma (de Gruchy, 1978; Krivit, Giebink and Leonard, 1979; 
McCulloch and Parker, 1979). When splenectomy has been performed for haematological disease such as lymphoma or thalassaemia major in children or adults, the commonest infecting organism is $S$. pneumoniae. Less frequently Neisseria meningitidis, Escherichia coli, Haemophilus influenzae, Staphylococcus aureus or malarial parasites are involved. There is increasing evidence that OPSI also occurs in otherwise normal adults, Strep. pneumoniae again being most frequently seen, with a mortality of 50-75\% (Krivit et al., 1979; Gopal and Bisno, 1977). Group B streptococci are increasingly recognized as human pathogens, especially in neonates. Infection in adults is seen particularly when there is co-existing genito-urinary pathology or diabetes mellitus (Bayer et al., 1976). They are usually of low pathogenicity, and have not to the authors' knowledge been previously reported as a cause of OPSI. In the present patient, however, they caused severe septicaemia with hypotension, jaundice and shock lung. This case adds further support to the growing evidence of OPSI in otherwise normal adults.

\section{Acknowledgments}

We thank Dr G. Laszlo for permission to report this case and Professor D. C. E. Speller for his assistance with its preparation.

\section{References}

Bayer, A.S., Chow, A.W., Anthony, B.F. \& Guze, L.B. (1976) Serious infections in adults due to Group B streptococci. American Journal of Medicine, 61, 498.

DE GRUCHY, G.C. (1978) In: Clinical Haematology in Medical Practice, 4th Edn. p. 603. Blackwell Scientific Publications, Oxford.

Gopal, V. \& BisNo, A.L. (1977) Fulminant pneumococcal infections in 'normal' asplenic hosts. Archives of Internal Medicine, 137, 1526.

Krivit, W., GiebinK, G.S. \& Leonard, A. (1979) Overwhelming post-splenectomy infection. Surgical Clinics of North America, 59, 223.

McCulloch, D.K. \& Parker, A.C. (1979) Overwhelming post-splenectomy infection caused by Streptococcus milleri. Journal of Infection, 1, 379. 